\title{
Tripterygium wilfordii Glycosides Upregulate the New Anti-Inflammatory Cytokine IL-37 through ERK1/2 and p38 MAPK Signal Pathways
}

\author{
Sen Wang, Rumeng Li, Suhui He, Lingge He, Hang Zhao, \\ Xiaohong Deng, and Zhangquan Chen
}

\begin{abstract}
Key Laboratory for Medical Molecular Diagnostic of Guangdong Province, Guangdong Medical University, Dongguan, Guangdong Province 523808, China
\end{abstract}

Correspondence should be addressed to Zhangquan Chen; czqhk@126.com

Sen Wang and Rumeng Li contributed equally to this work.

Received 14 July 2017; Revised 8 November 2017; Accepted 21 November 2017; Published 18 December 2017

Academic Editor: Raffaele Capasso

Copyright (C) 2017 Sen Wang et al. This is an open access article distributed under the Creative Commons Attribution License, which permits unrestricted use, distribution, and reproduction in any medium, provided the original work is properly cited.

As a Chinese traditional patent medicine, Tripterygium wilfordii glycosides (TWG) have been approved by the China State Food and Drug Administration (Z32021007) for autoimmune and inflammatory diseases. Application of TWG leads to significant decrease of the inflammatory cytokines, such as IL-6, IL-1 $\beta$, and TNF- $\alpha$. However, little is known whether TWG could regulate the antiinflammatory cytokines and what the mechanism is. Here, we found that TWG could induce the upregulation of IL-37 which is a new anti-inflammatory cytokine. Furthermore, the inhibitors of ERK1/2 and/or p38 MAPK pathways suppressed IL-37 expression induced by TWG, indicating that the two pathways took part in this process. In conclusion, TWG could upregulate the antiinflammatory cytokine IL-37 and ERK1/2 and p38 MAPK signal pathways were involved in the upregulation of IL-37 induced by TWG. The results showed that TWG had a potent activity on promoting the expression of IL-37, a new anti-inflammatory cytokine, which help further understanding the anti-inflammatory mechanism for the clinical application of TWG in therapy of diseases.

\section{Introduction}

During the years, Tripterygium wilfordii glycosides (TWG) have been demonstrated to be a powerful anti-inflammatory and immune modulatory drug $[1,2]$. TWG is a stable glycoside extracted from Tripterygium wilfordii Hook F (TwHF). In China, America, Korea, Japan, and other countries, TwHF is known as "LeiGongTeng" for treating autoimmune diseases [1-4]. Thus, TWG from TwHF has been approved by the China State Food and Drug Administration (Z32021007) for the routine treatment of Crohn's disease [5], rheumatoid arthritis (RA) [3], and ulcerative colitis [6]. TWG tablets can be orally administered. After entering the body, TWG is absorbed by passive diffusion. The organ with highest drug concentration is liver. And TWG is excreted mainly through feces and urine [7]. Treatment with TWG leads to significant decrease of the inflammatory cytokines. In ulcerative colitis, dextran sulfate sodium-induced IL-6 expression was inhibited by triptolide, the main extract from TWG [6]. Another study found that TWG inhibited the inflammatory mediators in interleukin-1 $\beta$-stimulated cells [8]. In another report, TWG could markedly reduce IL-6, IL- 8 , and TNF- $\alpha$ in serum of type II collagen-induced arthritis rats [9]. However, little is known whether TWG could regulate the anti-inflammatory cytokines and what the mechanism is. This problem is quite important to understand fully the treatment mechanism of TWG for autoimmune and inflammatory diseases.

Besides the inhibition of the expression of inflammatory cytokines, the mechanism of anti-inflammatory drugs often includes the effects on anti-inflammatory cytokines. Until recently, few attempts have been done for TWG on the influence of anti-inflammatory molecules. IL-37 is a newly named member of IL-1 family with powerful anti-inflammatory function. IL-37 contains five subtypes, IL-37a, b, c, d, and e, 
and IL-37 usually refers to IL-37b which is the largest subtype and shows important bioactive function. This gene is found to be expressed in a variety of tissues and cells, such as monocytes, natural killer (NK) cells, and epithelial cells [10, 11]. Here, we aim to discuss whether TWG could regulate a new anti-inflammatory cytokine, IL-37, and to elucidate the related mechanism. Researchers have found that IL-37 mediates a negative feedback mechanism to curb the excessive inflammation $[10,12,13]$. IL-37 could repress the proinflammatory cytokines expression, including IL-6, TNF- $\alpha$, and IL$1 \beta$ to reduce the inflammation response $[10,14-16]$. Most importantly, IL-37 was also involved in some inflammatory diseases that could be treated with TWG $[17,18]$, suggesting that TWG might have influenced IL-37 in those diseases. IL-37 expression has been found in human monocytes and human cell lines (such as THP-1, U937, A431, IMTLH, KG1, HL60, HPT-4, and NHDC). THP-1 is a suitable cell model for IL-37 research in many reports including the study which renamed IL-37 in 2010 in Nature Immunology [10]. So THP-1 cells were chosen in this study. The THP-1 cellular model for IL-37 expression was used as previously described [10]. We found that TWG upregulated IL-37 expression in THP-1 cells. Furthermore, ERK1/2 and MAPK were involved in the regulation.

\section{Materials and Methods}

2.1. Reagents and Cell. Anti-IL-37 monoclonal antibody, Goat Anti-Mouse IgG H\&L (FITC), and Mouse IgG1 were purchased from Abcam Corporation (Cambridge, UK). TWG (10 mg/tablet, Approval number Z35020431) was obtained in the form of tablets (Tripterygium wilfordii glycoside tablets) from Fujian Huitian Bio-pharma Co., Ltd. (GMP certificated, Fujian, China). The tablets were dissolved in the incubation medium at a final concentration of $5 \mathrm{mg} \mathrm{mL}^{-1}$ as stock solution. Inhibitor of extracellular signal-regulated kinase 1 (ERK1)/ERK2, U0126, and inhibitor of p38 MAPK, SB 203580, were from Cell Signaling Technology (Beverly, MA, USA). RNAiso Plus, Prime Script ${ }^{\mathrm{TM}}$ RT Master Mix and $\mathrm{SYBR}^{\circledR}$ Premix Ex Taq (Perfect Real Time) were from Takara Biotechnology Co., Ltd. (Dalian, China). Primers were synthesized by Sangon Biotech Co., Ltd. (Shanghai, China). eBioscience Fixation/Permeabilization Concentrate and Permeabilization Buffer $(10 \times)$ were from eBioscience Inc. (San Diego, CA, USA). Dimethyl sulfoxide (DMSO) and phorbol12-myristate 13-acetate (PMA) were from Sigma (St. Louis. MO, USA). THP-1 cell line was obtained from the Cell bank of Chinese Academy of Sciences, Shanghai, China. Cells culture reagents including 1640 medium and fetal bovine serum (FBS) were purchased from HyClone (Longan, UT, USA).

2.2. Cell Culture and Drugs Stimulation. THP-1 cells were maintained in RPMI-1640 medium at $37^{\circ} \mathrm{C}, 5 \% \mathrm{CO}_{2}$. The 1640 medium contained $10 \%$ fetal bovine serum (FBS), $100 \mathrm{U} \mathrm{mL}^{-1}$ penicillin, and $0.1 \mathrm{mg} \mathrm{mL}^{-1}$ streptomycin. Then, $1 \times 10^{6} \mathrm{~mL}^{-1}$ cells were planted in 48 -well culture plates and were treated with $50 \mathrm{ng} \mathrm{mL}^{-1}$ PMA for differentiation for $48 \mathrm{~h}$ [9]. The plastic-adherent cells were washed twice with sterile
Dulbecco's phosphate-buffered saline (PBS) and incubated with fresh RPMI 1640 medium containing 0.5\% FBS for $18 \mathrm{~h}$. On the basis of previous reports, TWG concentrations from 0 to $15 \mu \mathrm{g} \mathrm{mL}^{-1}$ were adopted to determine concentrationdependent effects of TWG on expression of IL-37 in the preliminary test. After incubation for different periods, the prepared cells were collected for analysis.

2.3. Quantitative Real-Time PCR. Total RNA was extracted by a Takara RNAiso Plus Kit. Then, cDNA was synthesized through a Prime Script RT Master Mix Kit. A SYBR Premix Ex Taq TM kit was used for Real-time quantitative PCR which was carried out on the ABI prism 7700 Sequence Detection System (Perkin Applied System, Foster City, CA, USA). The reaction was performed in $25 \mu \mathrm{L}$ reaction buffer. The reaction buffer was mixed with $12.5 \mu \mathrm{L}$ of $2 \times$ SYBR green Master Mix, $1 \mu \mathrm{L}$ cDNA, and $300 \mathrm{nM}$ primer. Here, GAPDH primers for PCR were as follows: F (ACCCAGAAGACTGTGGATGG) and R (TTCTAGACGGCAGGTCAGGT). Here, the subtype of IL-37 is IL-37b. The specific primers for real-time PCR were as follows: F (TTAGAAGACCCGGCTGGAAGCC) and R (AGATCTCTGGGCGTATGTAGT). PCR cycling conditions were $95^{\circ} \mathrm{C}$ for $2 \mathrm{~min}\left(\right.$ at $95^{\circ} \mathrm{C}$ for $5 \mathrm{~s}, 60^{\circ} \mathrm{C}$ for $30 \mathrm{~s}$, and $72^{\circ} \mathrm{C}$ for $30 \mathrm{~s}$ ) for 40 cycles. The thermal dissociation protocol was for the detection of the PCR product. Each experiment was done in triplicate.

2.4. Flow Cytometry Analysis of Intracellular IL-37. Cells were firstly incubated with fresh RPMI 1640 medium containing $0.5 \%$ FBS for $18 \mathrm{~h}$. Then, after pretreatment with TWG for $16 \mathrm{~h}$, the cells were collected and washed with ice cold staining buffer and were resuspended at $1.0 \times 10^{9} \mathrm{~L}^{-1}$. These cells were fixed with $100 \mu \mathrm{L}$ Fixation/Permeabilization working buffers and incubated for 20 minutes at room temperature in the dark. Next, $2 \mathrm{~mL} 1 \times$ Permeabilization working buffer was added to each tube. After washing twice, the cell pellets were resuspended with $100 \mu \mathrm{L} \mathrm{1x}$ Permeabilization buffer which contained $10 \%$ normal goat serum for serum blockage and were incubated for $1 \mathrm{~h}$ in the dark at room temperature. Then, cells were incubated with $1 \mu \mathrm{L}$ of IL-37 monoclonal antibody or mouse IgG1 isotype control antibody for $1 \mathrm{~h}$. Cells were washed with Permeabilization working buffer and incubated with the secondary antibody at 1/500 dilution for 30-60 min in the dark. Finally, the stained cells were acquired on the flow cytometer and the data were analyzed by BD FACSDIva software.

2.5. Analysis of Signal-Transduction Pathways. To discuss the mechanism involved in the over expression of IL-37 induced by TWG, the specific inhibitor for p38 MAPK or ERK1/2 pathway was used, respectively. After being pretreated with p38 MAPK inhibitor, SB203580 $\left(10 \mu \mathrm{mol} \mathrm{L}^{-1}\right)$, or ERK1/2 inhibitor, U0126 (5 $\left.\mu \mathrm{mol} \mathrm{L}{ }^{-1}\right)$, for $30 \mathrm{~min}$, macrophages from THP-1 cells were treated with TWG. The cells without pretreatment with SB203580 or U0126 were chosen as controls. After the drug stimulation for $12 \mathrm{~h}$, the cells were collected for RNA extraction for real-time PCR. After drug stimulation for $16 \mathrm{~h}$ the cells were collected for flow cytometry analysis. 


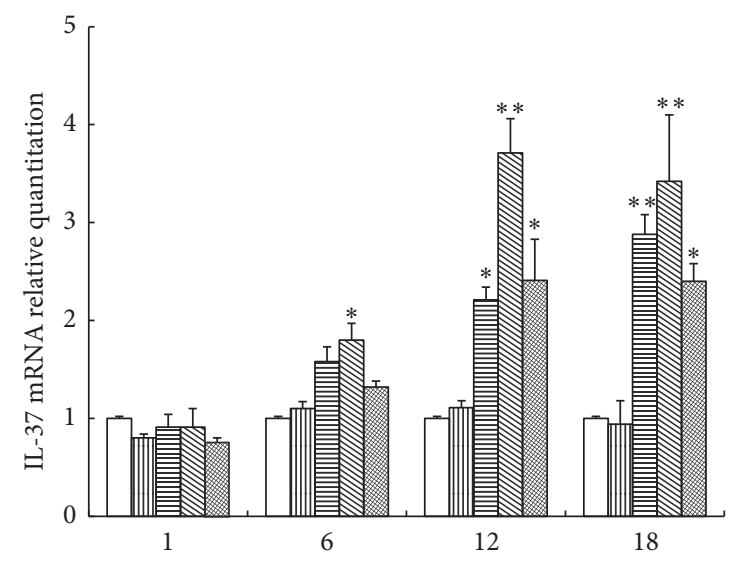

(h)

$$
\begin{aligned}
& \text { Control } \\
& \text { 四 } 1 \mu \mathrm{g} \mathrm{mL}^{-1} \\
& \text { 曰 } 5 \mu \mathrm{g} \mathrm{mL}^{-1} \\
& \text { ฐ } 10 \mu \mathrm{g} \mathrm{mL}^{-1} \\
& \text { 용 } 15 \mu \mathrm{gL}^{-1}
\end{aligned}
$$

Figure 1: IL-37 mRNA induced by TWG in macrophages was detected by quantitative real-time PCR. TWG represents Tripterygium wilfordii glycosides. * versus control, $p<0.05 ; * *$ versus control, $p<0.01$.

2.6. Statistics. All experiments were performed at three times. Data were expressed as means \pm SD and were analyzed with one-way analysis of variance (ANOVA) utilizing the SPSS version 13.0. Different groups were considered to be statistically significant at $p<0.05$.

\section{Results}

3.1. TWG Upregulated the Expression of IL-37 in THP-1 Cells. Macrophages were from THP-1 cells treated with PMA [10]. Here, TWG concentrations were $1 \mu \mathrm{gmL}^{-1}, 5 \mu \mathrm{gmL}^{-1}$, $10 \mu \mathrm{gmL}^{-1}$, and $15 \mu \mathrm{gmL}^{-1}$ for pretreatment of cells. After drug stimulation, total RNA was extracted from macrophages for CDNA synthesis and IL-37 mRNA was detected by qPCR. The results were presented in Figure 1. At 1h, IL-37 mRNA expression showed no obvious change for TWG at $1 \mu \mathrm{gmL}^{-1}$, $5 \mu \mathrm{gmL}^{-1}, 10 \mu \mathrm{gmL}^{-1}$ and $15 \mu \mathrm{gmL}^{-1}$. At $6 \mathrm{~h}, 12 \mathrm{~h}$, and $18 \mathrm{~h}$, $10 \mu \mathrm{gmL}^{-1}$ TWG showed the strongest capacity to enhance IL-37 expression. Furthermore, the levels of IL-37 expression elevated gradually with increasing incubation time from $1 \mathrm{~h}$ to $12 \mathrm{~h}$ in the presence of $10 \mu \mathrm{gL}^{-1}$ of TWG. When time was over $12 \mathrm{~h}$, the mRNA level began to decrease with further incubation, which indicated that the maximum IL-37 mRNA expression is reached.

In order to study more about the influence of TWG on expression of IL-37, IL-37 protein was examined by flow cytometry analysis. As shown in Figure 2, TWG upregulated the protein level of IL-37 in cells. IL-37 positive cells were approximately up to $82.3 \%$ in cells treated with $10 \mu \mathrm{g} \mathrm{mL}^{-1}$ of TWG with $16 \mathrm{~h}$ incubation. Only $17.7 \%$ IL-37 positive cells were detected in THP-1 cells without TWG treatment (negative control) (Figure 2(a)). The mean fluorescence intensity
(MFI) of cells treated with TWG was approximately 3.2-fold greater than that without TWG treatment (Figure 2(b)).

3.2. Effect of SB203580 and U0126 on TWG-Induced IL-37 Expression in THP-1 Cells. In order to further elucidate how TWG induces the upregulation of IL-37, p38 MAPK and ERK1/2 signaling pathways were investigated. The cells were treated with SB203580 or U0126 for $30 \mathrm{~min}$, respectively. Then the macrophages were incubated with TWG for $12 \mathrm{~h}$. The expression of IL-37 was measured by real-time PCR (Figure 3) and flow cytometry (Figure 4). The results showed that SB203580 or U0126 decreased markedly the expression of IL37 mRNA (Figure 3 ) and IL-37 protein (Figure 4). The results of qPCR showed that $\mathrm{U} 0126$ at $5 \mu \mathrm{mol} \cdot \mathrm{L}^{-1}$ almost completely abolished the expression of IL-37 mRNA induced by TWG in macrophages (Figure 3). The other inhibitor (SB203580) at $10 \mu \mathrm{mol} \cdot \mathrm{L}^{-1}$ reduced about $45 \%$ of IL-37 mRNA expression induced by TWG at $12 \mathrm{~h}$ (Figure 3 ).

As to the cytometry analysis, U0126 and SB203580 abolished most of IL-37 expression induced by TWG at $16 \mathrm{~h}$ of incubation in macrophages (Figure 4(a)). The mean fluorescence intensity (MFI) of cells treated with TWG + U0126 or TWG + SB203580 was approximately $50 \%$ of that of TWG group (Figure 4(b)).

\section{Discussion}

TWG is extracted from a traditional Chinese medicine herb, Tripterygium wilfordii Hook F (TwHF). TWG has been widely used in the treatment of inflammatory diseases $[1-4,19]$. Growing evidences prove that TWG could inhibit expression of inflammatory cytokines, such as IL- 6 , IL- $1 \beta$, and TNF- $\alpha$, to treat related diseases $[6,8]$. However, whether and how TWG influence the anti-inflammatory cytokines are still unclear. Interleukin 37 is one of the few anti-inflammatory cytokines in the interleukin family. An enormous amount of researches has confirmed that the proinflammatory cytokines, including IL- 6 , TNF- $\alpha$, and IL- $1 \beta$, play pivotal roles in the pathogenesis of the chronic inflammatory disorders [20, 21]. IL-37 also participates in diseases which could be treated by TWG, such as RA $[17,18]$. Thus, we speculated that TWG could regulate IL-37 expression besides the inhibition of inflammatory cytokines. In addition, THP-1-derived macrophages have been reported to be a valid model system for antiinflammatory drug screening [10]. Consequently, THP-1derived macrophages were used to assess the effect of TwFH extracts on expression of IL-37. Here, we found that use of TWG upregulated significantly the mRNA and protein of IL37. In light of the powerful anti-inflammatory function, this result might be a new mechanism for TWG to treat diseases.

To further discuss the mechanism involved in the upregulation of IL-37 induced by TWG, cell signaling pathways were investigated in this study. ERK1/2 signaling pathway and the MAPK signaling pathway have been identified as the major signaling pathways in inflammation [10]. TWG has been reported to influence ERK1/2 and MAPK signal pathways in diseases $[20,21]$. Thus, we hypothesized that TWG might regulate ERK1/2 and MAPK to influence IL-37 


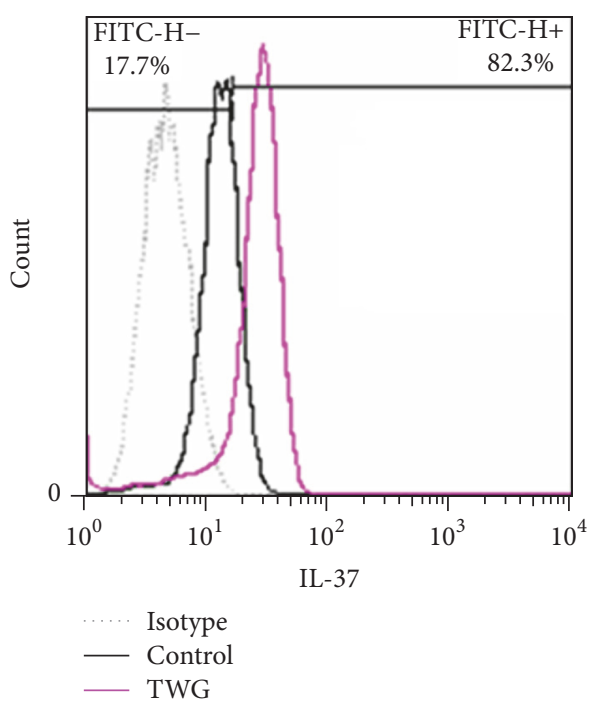

(a)

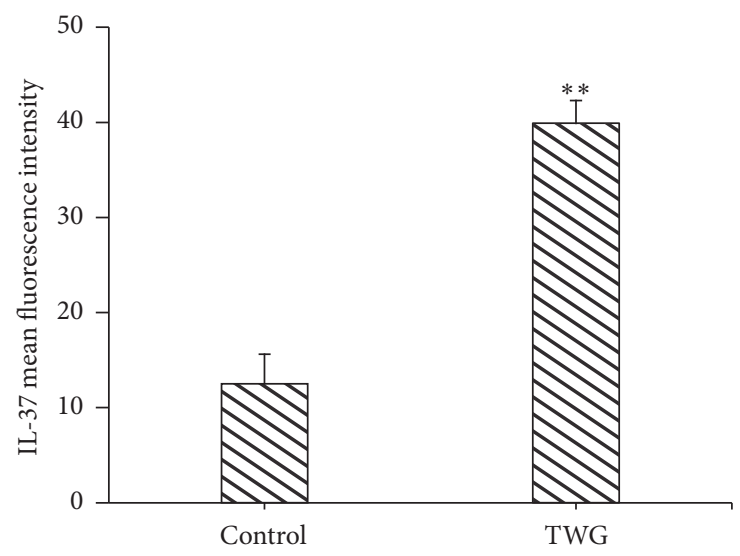

(b)

FIGURE 2: Effect of TWG on IL-37 protein in macrophages was examined by flow cytometry analysis. TWG represents Tripterygium wilfordii glycosides. $* *$ versus control, $p<0.01$.

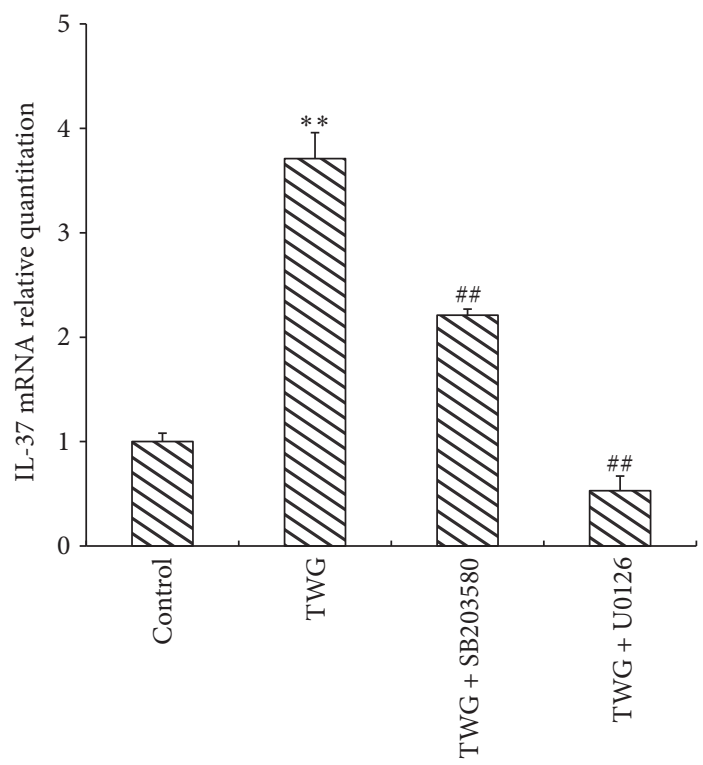

FIGURE 3: Effects of p38 inhibitor SB203580 and the ERK 1/2 inhibitor U0126 on TWG-induced mRNA expression of IL-37 were examined by real-time PCR in THP-1 cells. ** versus control, $p<0.01$; \# versus TWG, $p<0.01$.

expression. Our results showed that the p38 MAPK inhibitor (SB203580) and ERK1/2 inhibitor (U0126) could inhibit markedly the upregulation of IL-37 induced by TWG. It should be noted that the results could not be used to determine that $\mathrm{p} 38 \mathrm{MAPK}$ and ERK1/2 are the only pathways for TWG-induced IL-37. However, this paper does suggest that ERK1/2 and p38 MAPK pathways might be involved in the upregulation of IL-37 induced by TWG. In addition, in the work described by Imaeda et al. [14], NF-kB and AP-1 are involved in the induction of IL-37 by TNF- $\alpha$. However, TNF- $\alpha$ is an inflammatory mediator while TWG is an anti-inflammation drug. In the work described by
Sylvester et al. [22], a Tripterygium extract was found to inhibit the activity of NF-kB and AP-1. Thus, it seems that MAPK and ERK signals are more possible to take part in the induction of IL-37 by TWG. And the results did show that MAPK and ERK signals were involved in the TWG-induced IL-37 elevation. Thus, our study indicated a new possible mechanism for TWG to curb inflammation.

\section{Conclusion}

In this study, we found that TWG could induce the upregulation of IL-37 and the ERK1/2 and/or P38 MAPK pathways 


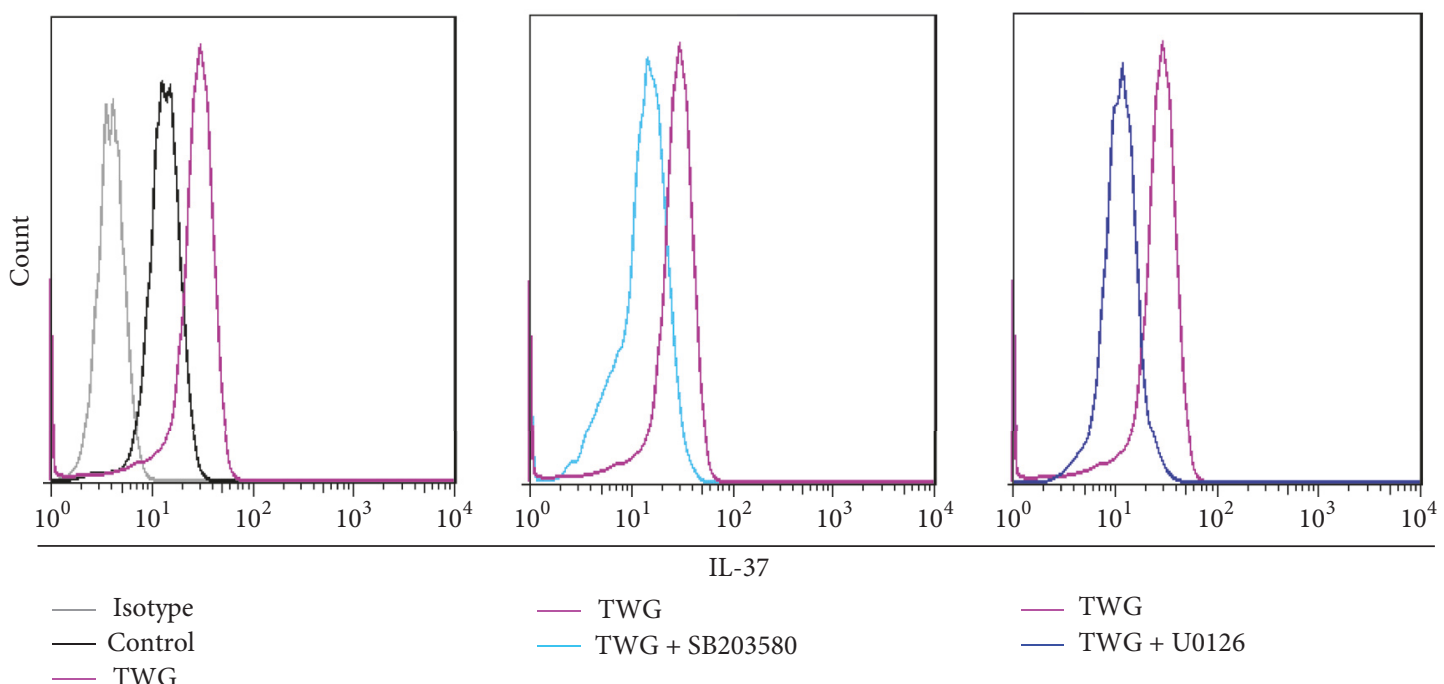

(a)

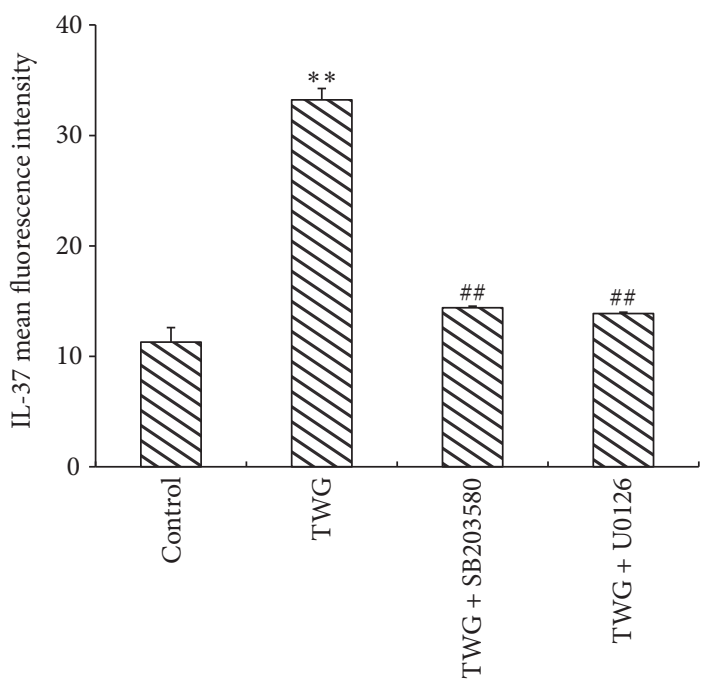

(b)

FIGURE 4: Effects of p38 MAPK inhibitor SB203580 and the ERK 1/2 inhibitor U0126 on the TWG-induced IL-37 protein were detected by flow cytometry in macrophages. TWG represents Tripterygium wilfordii glycosides. $* *$ versus control, $p<0.01$; \#\# versus TWG, $p<0.01$.

were involved in this process. Thus, our study indicated a new possible mechanism for TWG to curb inflammation.

\section{Additional Points}

Highlights. Tripterygium wilfordii glycosides upregulated the anti-inflammatory cytokine IL-37; ERK1/2 and p38 MAPK signal pathways were involved in the upregulation of IL37 induced by TWG. We showed a new anti-inflammatory mechanism for Tripterygium wilfordii glycosides.

\section{Conflicts of Interest}

There are no conflicts of interest to declare.

\section{Authors' Contributions}

Sen Wang and Rumeng Li contribute equally to this work, and they are co-first authors. Zhangquan Chen conceived and supervised the study; Zhangquan Chen designed experiments; Rumeng Li, Sen Wang, Suhui He, Lingge He, Hang Zhao, and Xiaohong Deng performed experiments; Rumeng Li, Sen Wang, Suhui He, Lingge He, and Zhangquan Chen analyzed data; Sen Wang and Zhangquan Chen wrote the manuscript and made manuscript revisions.

\section{Acknowledgments}

This study was funded by the Science and Technology Plan Project of Guangdong Province (2016A020215147), 
Medical Science and Technology Research Fund of Guangdong Province (A2016208, A2017605), the Social Science and Technology Development Project of Dongguan (2013108101051), Scientific Research foundation of Guangdong Medical University (Z2014002), Training Program of Innovation and Entrepreneurship for Undergraduates (201510571011, 201510571056), and National Natural Science Foundation of China (81302244).

\section{Supplementary Materials}

IL-37 is one of the few anti-inflammatory cytokines. TWG, a stable glycoside extracted from Tripterygium wilfordii Hook $\mathrm{F}$ (TwHF), is a powerful anti-inflammatory and immune modulatory drug. In this study, it is found that TWG could upregulate IL-37, and ERK1/2 and p38 MAPK pathways might be involved in the upregulation of IL-37 induced by TWG. This study indicates a new possible mechanism for TWG to curb inflammation. (Supplementary Materials)

\section{References}

[1] X. Tao and P. E. Lipsky, "The Chinese anti-inflammatory and immunosuppressive herbal remedy Tripterygium wilfordii Hook F," Rheumatic Disease Clinics of North America, vol. 26, no. 1, pp. 29-50, 2000.

[2] R. Han, M. Rostami-Yazdi, S. Gerdes, and U. Mrowietz, "Triptolide in the treatment of psoriasis and other immunemediated inflammatory diseases," British Journal of Clinical Pharmacology, vol. 74, no. 3, pp. 424-436, 2012.

[3] R. Goldbach-Mansky, M. Wilson, R. Fleischmann et al., "Comparison of Tripterygium wilfordii Hook F versus sulfasalazine in the treatment of rheumatoid arthritis: a randomized trial," Annals of Internal Medicine, vol. 151, no. 4, pp. 229-240, 2009.

[4] B. J. Chen, "Triptolide, a novel immunosuppressive and antiinflammatory agent purified from a Chinese herb Tripterygium Wilfordii Hook F,' Leukemia \& Lymphoma, vol. 42, no. 3, pp. 253-265, 2001.

[5] J. Ren, X. Wu, N. Liao et al., "Prevention of postoperative recurrence of Crohn's disease: tripterygium wilfordii polyglycoside versus mesalazine," Journal of International Medical Research, vol. 41, no. 1, pp. 176-187, 2013.

[6] H. Zhang and W. Chen, "Interleukin 6 inhibition by triptolide prevents inflammation in a mouse model of ulcerative colitis," Experimental and Therapeutic Medicine, vol. 14, pp. 2271-2276, 2017.

[7] J.-R. Lin, B. Lin, and H.-T. Song, "Research progress on in vivo pharmacokinetics of triptolide and celastrol," Chinese Traditional and Herbal Drugs, vol. 47, no. 3, pp. 528-532, 2016.

[8] A. Cai, S. Qi, Z. Su, H. Shen, W. Ma, and Y. Dai, “Tripterygium glycosides inhibit inflammatory mediators in the rat synovial RSC-364 cell line stimulated with interleukin-1 $\beta$," Biomedical Reports, vol. 3, no. 6, pp. 763-766, 2015.

[9] Y. Zhang, W. Xu, H. Li et al., "Therapeutic effects of total alkaloids of Tripterygium wilfordii Hook f. on collagen-induced arthritis in rats," Journal of Ethnopharmacology, vol. 145, no. 3, pp. 699-705, 2013.

[10] M. F. Nold, C. A. Nold-Petry, J. A. Zepp, B. E. Palmer, P. Bufler, and C. A. Dinarello, "IL-37 is a fundamental inhibitor of innate immunity," Nature Immunology, vol. 11, no. 11, pp. 1014-1022, 2010.
[11] D. Boraschi, D. Lucchesi, S. Hainzl et al., "IL-37: a new antiinflammatory cytokine of the IL-1 family," European Cytokine Network, vol. 22, no. 3, pp. 127-147, 2011.

[12] A.-M. Bulau, M. F. Nold, S. Li et al., "Role of caspase-1 in nuclear translocation of IL-37, release of the cytokine, and IL-37 inhibition of innate immune responses," Proceedings of the National Acadamy of Sciences of the United States of America, vol. 111, no. 7, pp. 2650-2655, 2014.

[13] L. Ye, L. Ji, Z. Wen et al., "IL-37 inhibits the production of inflammatory cytokines in peripheral blood mononuclear cells of patients with systemic lupus erythematosus: Its correlation with disease activity," Journal of Translational Medicine, vol. 12, no. 1, article no. 69, 2014.

[14] H. Imaeda, K. Takahashi, T. Fujimoto et al., "Epithelial expression of interleukin-37b in inflammatory bowel disease," Clinical \& Experimental Immunology, vol. 172, no. 3, pp. 410-416, 2013.

[15] G. Pan, P. Risser, W. Mao et al., "IL-1H, an interleukin 1-related protein that binds IL-18 receptor/IL-1Rrp," Cytokine, vol. 13, no. 1, pp. 1-7, 2001.

[16] E. N. McNamee, J. C. Masterson, P. Jedlicka et al., "Interleukin 37 expression protects mice from colitis," Proceedings of the National Acadamy of Sciences of the United States of America, vol. 108, no. 40, pp. 16711-16716, 2011.

[17] L. Yang, J. Zhang, J. Tao, and T. Lu, "Elevated serum levels of Interleukin-37 are associated with inflammatory cytokines and disease activity in rheumatoid arthritis," APMIS-Acta Pathologica, Microbiologica et Immunologica Scandinavica, vol. 123, no. 12, pp. 1025-1031, 2015.

[18] M. Wang, B. Wang, Z. Ma et al., "Detection of the novel IL-1 family cytokines by QAH-IL1F-1 assay in rheumatoid arthritis," Cell Mol Biol (Noisy-le-grand), vol. 62, pp. 31-34, 2016.

[19] Y. Liu, S. Tu, W. Gao et al., "Extracts of Tripterygium wilfordii hook $\mathrm{F}$ in the treatment of rheumatoid arthritis: a systemic review and meta-analysis of randomised controlled trials," Evidence-Based Complementary and Alternative Medicine, vol. 2013, Article ID 410793, 11 pages, 2013.

[20] T. Tanaka and T. Kishimoto, "Targeting interleukin-6: all the way to treat autoimmune and inflammatory diseases," International Journal of Biological Sciences, vol. 8, no. 9, pp. 1227-1236, 2012.

[21] L. He, Z. Liang, F. Zhao, L. Peng, and Z. Chen, "Modulation of IL-37 expression by triptolide and triptonide in THP-1 cells," Cellular \& Molecular Immunology, vol. 12, no. 4, pp. 515-518, 2015.

[22] J. Sylvester, A. Liacini, W. Q. Li, F. Dehnade, and M. Zafarullah, "Tripterygium wilfordii hook F extract suppresses proinflammatory cytokine-induced expression of matrix metalloproteinase genes in articular chondrocytes by inhibiting activating protein- 1 and nuclear factor- $\kappa \mathrm{B}$ activities," Molecular Pharmacology, vol. 59, no. 5, pp. 1196-1205, 2001. 


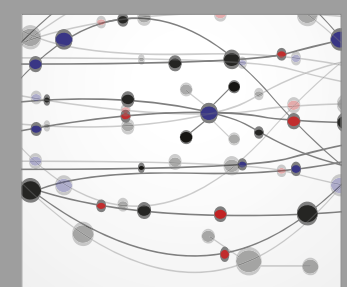

The Scientific World Journal
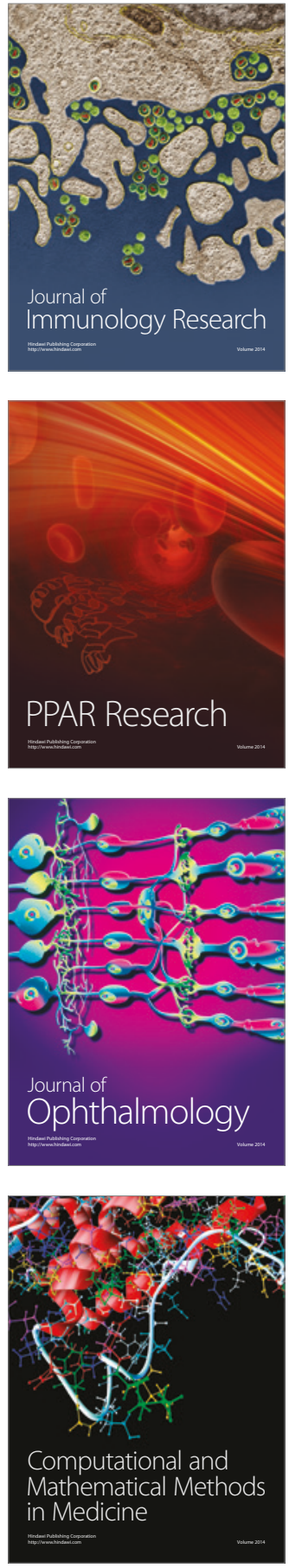

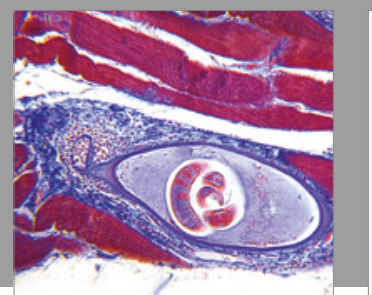

Gastroenterology Research and Practice
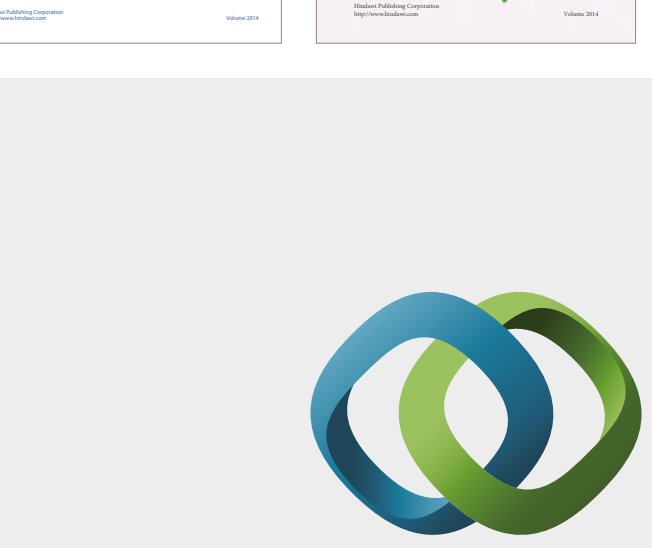

\section{Hindawi}

Submit your manuscripts at

https://www.hindawi.com
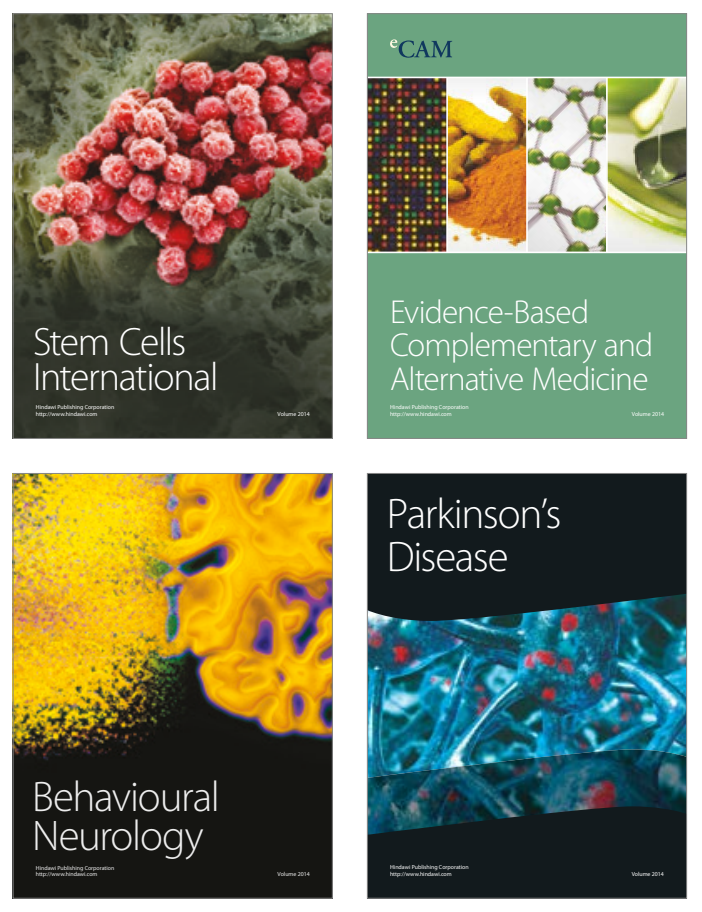
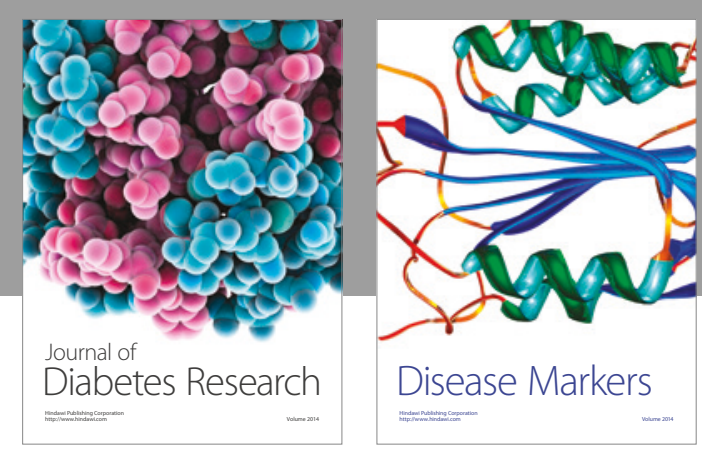

Disease Markers
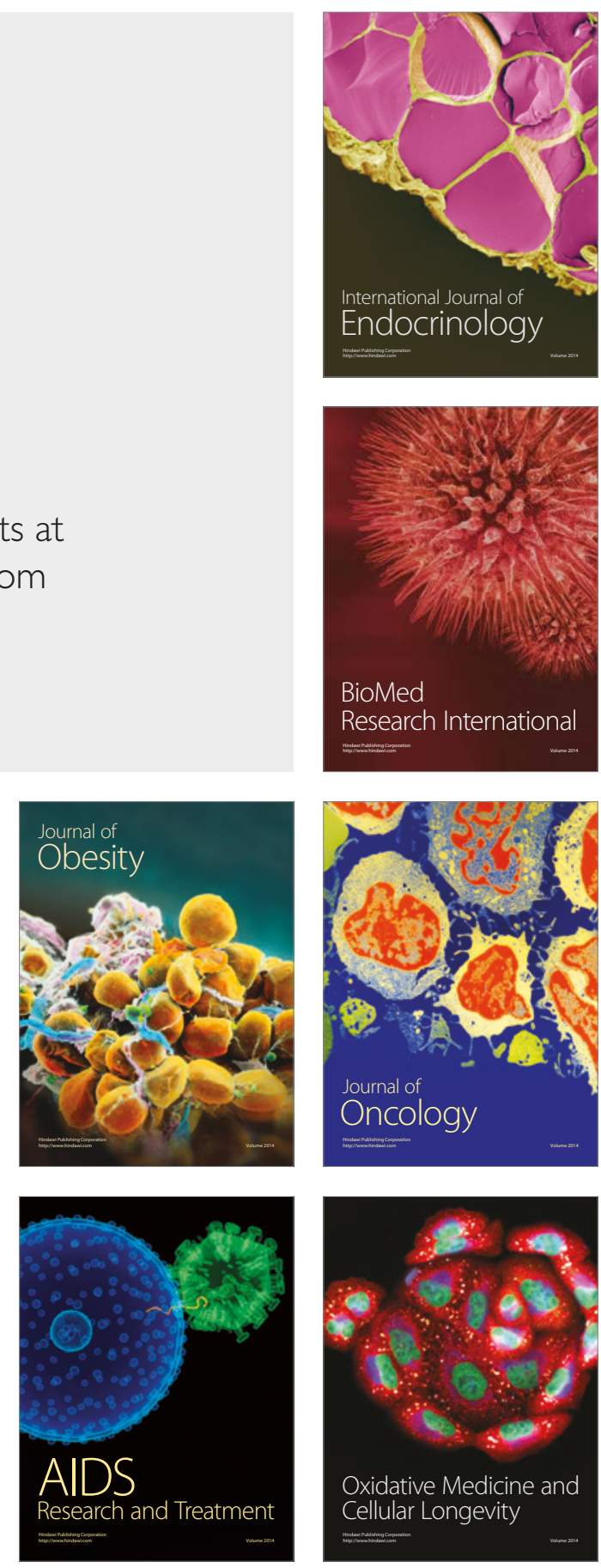